Article

\title{
Increasing the Yield of Lactuca sativa, L. in Glass Greenhouses through Illumination Spectral Filtering and Development of an Optical Thin Film Filter
}

\author{
Jacqualine A Thomas*, Mikhail Vasiliev@, Mohammad Nur-E-Alam® and Kamal Alameh \\ Electron Science Research Institute, School of Science, Edith Cowan University, 270 Joondalup Drive, \\ Joondalup, WA 6027, Australia; m.vasiliev@ecu.edu.au (M.V.); m.nur-e-alam@ecu.edu.au (M.N.-E.-A.); \\ k.alameh@ecu.edu.au (K.A.) \\ * Correspondence: j.thomas@ecu.edu.au
}

Received: 28 March 2020; Accepted: 28 April 2020; Published: 5 May 2020

\begin{abstract}
With the increase in world population, the continued advances in modern greenhouse agriculture and plant growth practices are expected to help overcome the global problem of future food shortages. The next generation greenhouse design practices will need to address a range of issues, ranging from energy and land use efficiency to providing plant-optimized growth techniques. In this paper, we focus on investigating the optimum irradiation spectra matched to the lettuce species (Lactuca sativa, L.), commonly grown in greenhouse environments, in order to develop low-emissivity glass panes that maximize the biomass productivity of glass greenhouses. This low-emissivity glass passes the solar spectral components needed for crop growth, while rejecting other unwanted radiations. This could potentially lead to significant energy savings and other beneficial effects related to greenhouse climate control, in a range of climates. The experimental results show that substantial biomass productivity improvements in lettuce (up to approximately $14.7 \%$ ) can be attained by using spectrally optimized illumination, instead of white light illumination. This optimized wavelength is then demonstrated as being used to develop an advanced metal-dielectric thin-film filter that produces the optimized illumination spectrum when exposed to sunlight.
\end{abstract}

Keywords: greenhouse agriculture; Lactuca sativa; spectral optimization; energy-efficiency

\section{Introduction}

In 2050, the world could potentially be facing a food crisis. According to the United Nations Food and Agriculture Organisation (UN FAO), the world population is projected to be $39 \%$ above that of 2006, by 2050. However, several of the most food-insecure countries are projected to have much larger population increases, e.g., Niger (the country with the highest total fertility rate in the world), is projected to grow from 14 million in 2006 to 58 million in 2050, a 4.3-fold increase. Almost all of these countries have been in nearly perennial food insecurity for several decades [1]. A possible way forward has been proposed recently in The Lancet [2], which is to consider amending the diet of the population, to improve nutrition, but also ensure environmental sustainability. According to the EAT-Lancet Commission, food systems can provide a healthy reference diet for an estimated global population of about 10 billion people by 2050 and remain within a safe operating space.

However, even small increases in consumption of red meat or dairy foods would make this goal difficult or impossible to achieve [3]. Within the proposed reference diet of the EAT-Lancet Commission, there is a strong focus on vegetables. All vegetables make up approximately $200-600 \mathrm{~g} /$ day of the macronutrient intake of a person on the proposed reference diet [3]. Of this range, approximately $100 \mathrm{~g} /$ day is comprised of dark green vegetables [3]. These "dark green vegetables" are a rather broad 
category of plant species, loosely defined by their leaf coloration type. Spinach, and several lettuce sub-species, also fit into this category.

Advanced growth technologies, such as innovative greenhouse production methods, are widely expected [4-8] to provide the practical solutions to these issues. Despite the growing body of knowledge and numerous literature reports detailing the beneficial effects of using some particular types of spectrally-optimized artificial irradiation profiles on plant growth, it becomes apparent, at present, that new multi-disciplinary approaches need to be developed for future greenhousing, combining the advantages of natural, filtered natural, artificial, and filtered-artificial irradiation regimes with energy-efficiency improvements.

With this in mind, it will be beneficial to the world population along with the agriculture industry, if green vegetables, such as lettuce (Lactuca sativa, L.), could be efficiently mass-produced with minimal energy use, whilst maintaining the nutritional quality, simultaneously with achieving increases in the biomass growth productivity. To achieve this, harvesting solar energy using highly transparent solar photovoltaic (PV) windows has been considered. Solar PV is one of the most promising approaches to improving energy-efficiency in greenhouses, and can be combined synergistically with spectral shaping of the incoming solar radiation using thin-film filters, which can also reduce the solar heat gain (in hot climates), and help keep the heat trapped inside (in colder climates), whilst having the optical transmission peaks tuned to the photosynthetically- or yield-sensitive absorption bands of plant tissues.

Lactuca sativa L. was chosen as the sample plant, as it is a quantitative long-day plant at high temperatures and a day-neutral plant at low temperatures [9]. This allowed production yields to be maximized, by controlling both the irradiation timing and the ambient temperature so that when the length of day exceeds the length of the night that the plant detects, the plant will not bolt, but will instead grow optimally. Additionally, the choice of plant type was also limited to available optical intensity levels at plant leaf surfaces, due to using LED sources of low power consumption and the necessity of providing an LED irradiation area as uniform as possible. High temperatures are considered to be approximately room temperature, $18-20^{\circ} \mathrm{C}$ minimum [8]. Accordingly, the grow tents were kept at a temperature of approximately $21.4{ }^{\circ} \mathrm{C}$. Lactuca sativa $\mathrm{L}$. will "bolt", that is, flower too soon, when the temperature is not kept high and steady [9]. Bolting effects were not observed in our growth experiments. Increasing the length of day from that provided by the sun, would be necessary (throughout most or all locations based in moderate latitudes) for lettuce plants to grow optimally [7-9]. For greenhouse locations within or near the Arctic Circle, a summer growth season will include naturally-occurring long daylight conditions; however, the natural irradiation intensity will be much weaker, even near midday, compared to more temperate or hot climates. Utilizing artificial lighting, for example, LED lighting, is then either highly desirable, or necessary, and energy-efficiency considerations are of essential importance for large scale agricultural production [10-13], whether the greenhouse facilities are located in moderate or cold climates.

Greenhouse materials cover a broad range, from simple glass or plastics, to building integrated or building-applied advanced photovoltaic (BIPV or BAPV, respectively) greenhouse components. This has resulted in the emergence of a new field, Agrivoltaics [12]. The idea of a photovoltaic greenhouse is that photovoltaic modules are placed in various positions and configurations onto a greenhouse roof or walls, and the incident sunlight is used to not only grow the plants, but also generate electricity when the incident sunlight strikes the PV modules [12]. The problem with all or most photovoltaic greenhouses reported up to date, lies in the problem of PV modules taking up areas on the building envelope of the greenhouse, thus strongly shading the plants. These shading effects can also potentially lead to the undesirable bolting of plants [14]. Even the recently demonstrated organic advanced luminescent solar concentrator (LSC) technologies using luminescent solar cell technologies with conventional silicon-based PV, which are beginning to be adapted to greenhouses, are not free of this shading problem [15]. We attempt to remove this shading problem through combining high 
transparency spectrally selective thin-film technologies with all-inorganic glass and modern results in advanced agricultural practices, for example, spectrally optimized LED lighting.

During this study, we concentrated on growing lettuce plants in soil, within controlled grow tents, not dissimilar to growth chambers. The research aim was to determine which wavelength range(s) of visible electromagnetic radiation from LED light sources outputting narrow wavelength ranges, are required to obtain optimum biomass productivity in lettuce plants. This result would then be translated into research aimed at developing thin-film optical filters for application to glass for future proposed use on the roof or walls of a greenhouse. The thin-film optical filter will pass through only the solar spectral components required for optimum biomass growth of the sample plant (lettuce). Alternatively, the same thin-film components can be used to filter a range of broad-bandwidth artificial light sources used at nighttime, to further boost the overall greenhouse productivity, simultaneously with reducing the heat load inside the greenhouse. The results can then be translated into customized thin-film filter which can then be used to filter the light spectra of any conventional broad-spectrum source. Alternatively, these filters on glass substrates could be used as components of the Electron Science Research Institute's (ESRI) recently developed transparent solar energy harvesting windows $[16,17]$.

Biomass was chosen as the control factor, as it is an indicator of how well a plant has photosynthesized incoming sunlight to produce energy for growth and grown mass accordingly $[9,18]$. The results obtained with spectrally optimized irradiation show an approximate $14 \%$ increase in dry biomass yield improvement when using the reduced spectrum blue, red, and far-red LED grow tent, compared to the white LED control grow tent. Additionally, an approximate $6 \%$ increase in biomass yield was demonstrated when using the reduced spectrum blue and red grow tent. This is a significant increase in biomass productivity, given that the available light for photosynthesis has been decreased in both grow tents in comparison to the white LED control tent. The present research presents a viable way forward towards the next generation of spectrally-selective greenhouses. We also point out that genetic measurements of the harvested lettuce plants were not taken in this experiment. Future experiments will consider the question as to whether the lettuce that was grown under the LEDs and also under the thin film filter with sunlight as the source, are suitably nutritionally edible.

In order to lengthen the photoperiod per day in a greenhouse, many PV greenhouses use the electricity generated to power artificial lighting systems [10]. This is not efficient overall, since a product of three conversion efficiency factors (the PV efficiency, the battery storage efficiency, and the electrical-to-optical conversion efficiency applicable to the light sources) will require further consideration $[11,16]$. Even if the most efficient electronic systems and LED components were used, the result is still not particularly efficient.

These considerations suggest that using passive optical components for filtering the natural sunlight spectrum is a more efficient approach to providing improved energy consumption, in comparison to using the most efficient of LED lighting systems. The energy balance improvements potentially offered by the use of these spectrally selective filters could be further enhanced if the films are integrated into transparent solar windows [16]. Regarding future research efforts, the investigation of the combined beneficial effects on both the plant growth and the greenhouse energy-efficiency in outdoor installations will be of interest. This would have to be researched using a combination of natural lighting optimally filtered through solar window coatings, and spectrally tuned artificial lighting systems or filtered white artificial lighting systems.

\section{Background, Prior Studies and Experimental Methodologies}

Research already conducted in this area has reported increased biomass productivity when using combinations of various LED light sources in comparison to fluorescent lamp-based lighting as the control group [18]. Additionally, using different combinations of LED lights at various stages of lettuce plant growth has also been reported to increase dry biomass [6]. Currently, the published literature sources have not investigated using white LED lighting systems as a control group, and comparing the 
biomass increases under various different light conditions to this control. In this paper, we particularly correlate illumination spectra that comprise far-red wavebands to the biomass of the lettuce plants.

By adjusting the outputs of the LED lighting systems to approximate within the technical limits applicable to the maximum light source outputs at each wavelength, the absorption of naturally occurring sunlight as far as possible intercepted by the leaves during each 24-hour period within either the full spectrum of a combined-LED source, or the spectral radiation components of discrete LED sources, quantitative characterization of irradiating light is enabled. Then, the biomass results could be considered comparable to experiments conducted in a greenhouse employing glass coated with thin-film filters designed to shape the spectra accordingly. The subsequent sections describe the details of our approach, methodologies, experimental activities and results. The results were able to be used to design a customized metal-dielectric thin-film optical filter suitable for improving plant productivity concurrently with being suitable for integration into energy-generating solar windows.

The experiments were conducted using three Heliospectra LX602C LED light sources supplied by Heliospectra (Göteborg, Sweden). The core hypothesis was that improved lettuce plant productivity could be achieved by focusing on each of the three important regions of illumination, that is, blue, red, and far-red (visible region of NIR). This was when compared to broad-band energy-efficient LED artificial lighting emulating a white spectrum, for lettuce. This hypothesis was based on the existing body of literature, in which the existence of photosynthetic productive spectral ranges, where the importance of far-red, has been reported [18-21]. However, no direct growth productivity comparisons are available for Lactuca sativa, L., in which white LED sources were used as a reference in regard to biomass. While the far-red light is not absorbed sufficiently strongly in plants to be important for photosynthesis, other phenomena (e.g., photomorphogenetic phenomena in plants) depend on this range of wavelengths-thus, the biomass production can still benefit from far-red supplementary lighting.

Due to a much wider market acceptance of white LED lighting sources compared to the narrow-linewidth red or blue LEDs used in specialized applications, it can easily be foreseen that the use of cost-efficient and economic white LED lighting of high optical power output per Watt of consumed energy will continue to grow in commercial greenhouse environments. The effect of different wavelengths on the relative efficiency of photosynthesis per incident photon for a single leaf in low-light conditions reported in Reference [22] and illustrated in Reference [23], also suggests the potential benefits of broad-band illumination versus using the narrow-linewidth discrete sources. The fact that almost all plant life has evolved under the naturally-occurring sunlight (a very broad-bandwidth light source) points to the potential usefulness of broad-bandwidth sources in greenhousing, even though white LEDs cannot match natural sunlight very closely for photosynthesis stimulation. A recent review article [24] provided some examples showing how the combinations of LED sources can approximately match the natural sunlight to ensure the growth and development of photosynthetic organisms, and how the changes in intensity and wavelength can manipulate plant metabolism. The multiple benefits of using LED sources in greenhousing, compared to high intensity discharge lamps, such as metal halide and high-pressure sodium lamps, are also discussed in Reference [24].

Additionally, ESRI's prior preliminary experiments growing capsicums in growth tents using Heliospectra light sources, having the combinations in individual grow tents of (blue and far-red), and (red and far-red) compared to white LED illumination, led to the finding that far-red was a tangible and significant growth stimulator. This also led to observing up to a $46 \%$ increase in wet weight between the red, far-red light condition, compared to the white light condition (for capsicums). As it is known in the literature that blue LED light is also required for optimum plant growth resulting in optimum biomass [6,18], the experiment was designed to compare one light treatment of blue and red LED light to another light treatment of blue, red, and far-red LED light and both treatments compared to the control of white LED light.

The Heliospectra LED light sources are programmable to emit wavelengths of light at $448 \mathrm{~nm}$ (visible blue spectrum), $666 \mathrm{~nm}$ (visible red spectrum), $736 \mathrm{~nm}$ (visible far-red spectrum) and the 
broader spectrum white LED light (5700K visible white light, with peaks at approximately $446 \mathrm{~nm}$, $534 \mathrm{~nm}$, and $625 \mathrm{~nm}$ ). The wavelengths can be tailored to emit in different combinations at varying power output (referred to as an 'intensity' setting on the Heliospectra system, however not actually an intensity measurement value). The spectral plots of the different light treatments in each grow tent are shown in Figure 1. The spectral plots were measured using a fiber visible spectrometer (Ocean Optics, USB 2000, calibrated according to the manufacturer instructions) configured for diffused reflectance spectrum measurements, with two measurements taken. Point 1 directly under the LED in the center of each grow tent at plant height approximately $130 \mathrm{~mm}$ above the pot, point 2 at the right of the tent in the center of the LED light also at plant height approximately $130 \mathrm{~mm}$ above the pot. The averages of the two measurements are plotted in Figure 1a-c.
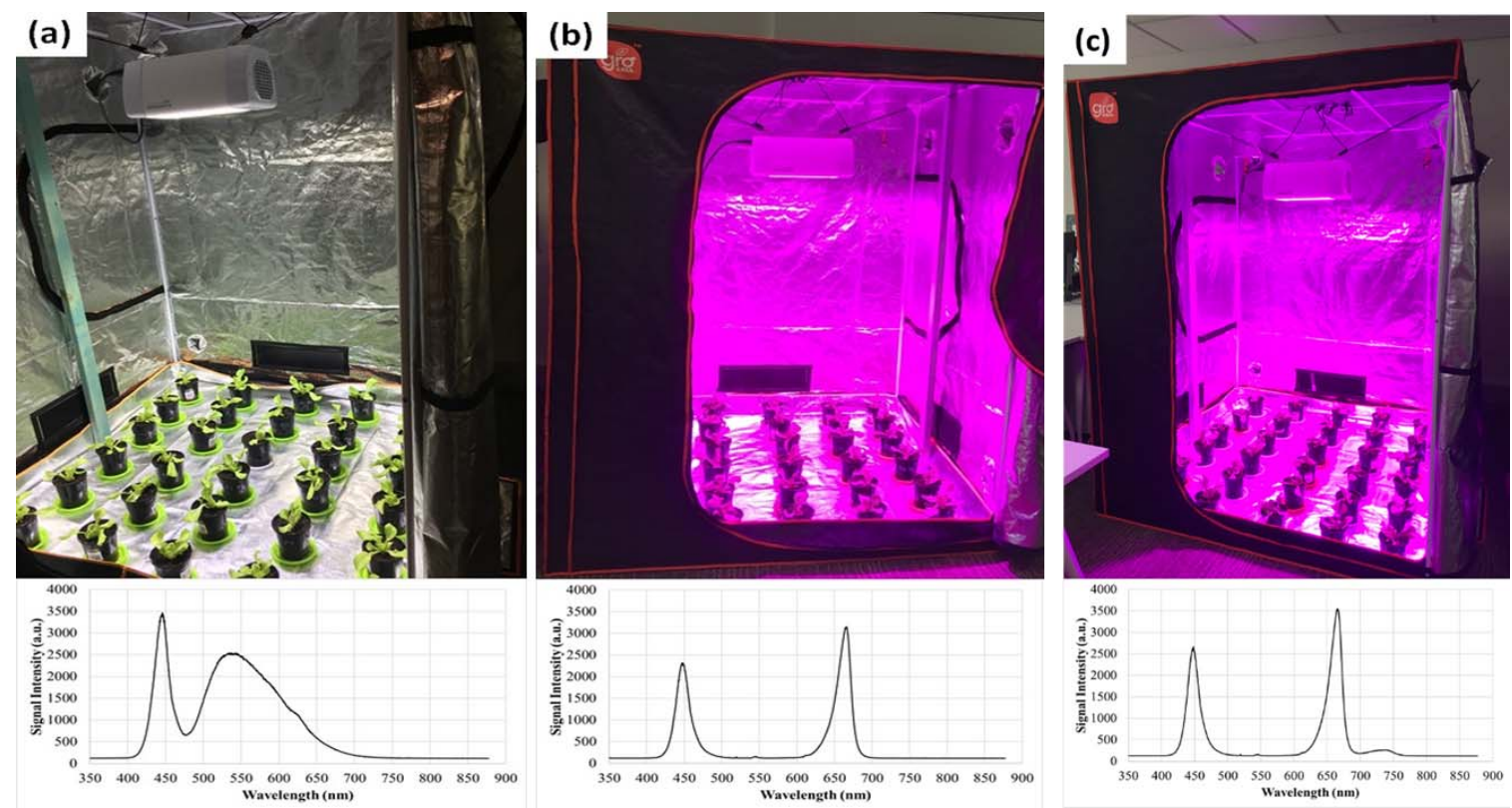

Figure 1. Illumination spectra used in each grow tent. (a) Grow Tent 1 has a white control spectrum of 1000 Heliospectra 'intensity' setting. (b) Grow Tent 2 has a blue and red control spectrum of blue 1000 Heliospectra 'intensity' setting, and red 458 Heliospectra 'intensity' setting. (c) Grow Tent 3 has a blue, red, and far-red control spectrum of blue 1000 Heliospectra 'intensity' setting, red 458 Heliospectra 'intensity' setting, and far-red 1000 Heliospectra 'intensity' setting. The photos were taken at nine days after planting from a seedling.

The spectrum power ('intensity' reading) values were determined after calibrating the Heliospectra LED light source to the power output and distance from the LED light for the various wavelengths. A detailed description of the power calibration procedure is set out in the Supplementary Information, where the wavelength calibration procedure used to periodically re-check the performance of the Ocean Optics spectrometer is also described.

\section{Correlating Source Power Density to the Incident Energy Available to Plants over Daytime Irradiation Periods}

We used the spectral intensity distribution data measured for the optical compound-source output from the Heliospectra, to derive the full width half maximum (FWHM) partial-source LED bandwidth data at each of the central (peak) LED wavelengths, for each of the colors (448 nm, $666 \mathrm{~nm}$ and $736 \mathrm{~nm}$ ) generated by the Heliospectra system. These data and parameters are needed to correlate the total optical energy received by lettuce leaves (each $1 \mathrm{~cm}^{2}$, per each artificial irradiation daytime duration), to the naturally occurring solar irradiation conditions. This correlation cannot be made to exactly mimic the daily solar energy absorption, but this is not necessary, due to the intrinsic 
variability of natural sunlight, the impracticality of using large-area solar simulators in greenhouse experiments, and also due to the output power range limitations applicable to all available LED sources. The energy intercepted by leaves from the natural solar irradiation can be evaluated (for peak sunshine conditions) by numerically integrating the standard (AM1.5G) spectral power density distribution, over the spectral limits corresponding to the central wavelength of each LED source and its FWHM boundaries, and by multiplying the resulting flux density (per each source, in $\mathrm{W} / \mathrm{m}^{2}$ ) by the typical peak-equivalent sunshine hours (PSH) value. The results of these calculations then approximate the total energy intercepted by leaves, per unit area, per unit irradiation time (or per average day, in the case of natural sunlight), thus, allowing adjustment of the LED source driver settings and choice of the most practical source-to-plant distance, together with the artificial daylight duration, to mimic within the limitations, the energy available to plants from natural sunlight daily. Considering the natural, weather and season-related variability of natural sunlight, being able to correlate the available energy within each discrete spectral band to within approx. the same order of magnitude (compared to the peak-time, clear-day sunshine conditions), will then approximately model the natural illumination. Natural sunlight also varies by about one order of magnitude in flux density, over the course of a typical sunny day. The visual appearance of the white can also confirm the suitability of white LED for mimicking natural sunlight LED illumination background at the floor level in tens (Figure 1a). This is also taking into consideration that the human eye, being an organ of vision, is a much more sensitive detector of the spectral intensity distribution of light, compared to at least the plant leaves, and the discrete-source artificial lighting background mimics sunlight in terms of its visual appearance.

The industrial use of white LED light sources in greenhousing is expanding continually at present, and the results reported in relation to plant growth experiments show that white LED irradiation did, in fact, lead to observing biomass growth improvements, compared to natural sunlight [25]. Thus, we believe that this type of broad-band light source can be used as a reference against which to compare the growth results obtained in other growth tents with more discrete-source, narrow-band combination-source spectra. The superior long-term stability of its spectral distribution and flux density (measured at leaf level during the 18-h daily irradiation period) is also a very important factor in running quantitative biomass-growth experiments. Moreover, obtaining improved biomass productivity and/or growth rate results, using more narrow-band lighting, can indicate that using a combination of filtered natural and filtered artificial (broad-band, e.g., metal-halide) light sources could improve greenhouse productivity. This would be done through designing a thin-film filter that modifies the natural sunlight to the spectral specifications close to those of the LED source that were identified by the experiment as providing optimum biomass results for lettuce. Furthermore, this would need additional confirmation in experiments which will be conducted at the solar window greenhouse to be constructed at Murdoch University (Perth, WA, Australia), in 2020. The scope of this study is limited to growth tent experiments, which compare the uses of artificial broad-band (white LED) and artificial narrow-band (a combination of other LEDs).

A custom-built spectral calculator (spectral integrator) program (designed by M. Vasiliev, ESRI, Edith Cowan University) was used to numerically integrate the standard NREL's data [26] for the sunlight power density distribution, using the spectral characterization data (integration limits) obtained for each artificial (LED) source. For the far-red LED source, the peak sunshine-equivalent natural-sunlight irradiation intensity was calculated to be $52.489 \mathrm{~W} / \mathrm{m}^{2}$ (rounded to three decimal places ( 3 d.p.)). The peak-equivalent daily sunshine conditions last for about 4.5 hours per day (4.5 PSH is a conservative estimate derived from averaging the peak-equivalent sunshine hours for Australia and the US $[27,28])$. Using these data for the PSH and for the target power density to be achieved at plant leaf, the daily energy available to natural-sunlight driven processes incident at the far-red wavelength selected, equates to about $850.328 \mathrm{~kJ} / \mathrm{m}^{2} /$ day. In comparison, full power (1000 'intensity' far-red setting) of the Heliospectra at approximately $1.4 \mathrm{~m}$ from the LED source, over an 18-hour day provides only $96.798 \mathrm{~kJ} / \mathrm{m}^{2} /$ day. That is, the Heliospectra will need to be kept at full power over an 18-hour day and will still provide a fraction of the far-red energy, compared to what the sun provides, at this 
source-to-plant distance, on a clear sunny day. Thus, achieving any measurable biomass productivity improvements attributable (at least in part) to use this relatively low-power LED source can suggest that a proposed thin-film filter design for glass greenhouses should aim at maximizing transmission of light in the far-red range.

The red and blue wavelengths were also considered in the same manner, yielding the blue power density to be achieved as approximately $28.519 \mathrm{~W} / \mathrm{m}^{2}$. This equates to about $462 \mathrm{~kJ} / \mathrm{m}^{2} /$ day, considering 4.5 PSH and a clear sunny day. Comparing this to the full power (1000 'intensity' setting) of the Heliospectra at the initial plant height, being approximately at $1.4 \mathrm{~m}$ from the LED source, over an 18-hour day this would provide approximately $146.5 \mathrm{~kJ} / \mathrm{m}^{2} /$ day. That is, the full power setting of blue will need to be used. Therefore, it was determined to maintain the blue source also at full power, rather than drop the height of the LED source, as red LED energy output still needed to be considered. In a similar manner, the red power density to be achieved was approximately $33.4 \mathrm{~W} / \mathrm{m}^{2}$. This equates to approximately $541.14 \mathrm{~kJ} / \mathrm{m}^{2} /$ day. Comparing this to the full power (1000 'intensity' setting) of the Heliospectra at the initial plant height, being approximately at $1.4 \mathrm{~m}$ from the LED source, over an 18-hour day, this would provide approx. $1160 \mathrm{~kJ} / \mathrm{m}^{2} /$ day. That is, at the full power setting of red LED, the Heliospectra at a height from the ground of $1.53 \mathrm{~m}$, will provide more light energy in the red visible range than the sun on an average clear sunny day. This issue was overcome by reducing the intensity of the red source from its full power to approximately half power as there was approximately 2.1 factor increase in the 18-h daily energy from the Heliospectra for red LED, in comparison to the output of the sun over a 4.5 PSH day, in the same wavelength range defined by the source FWHM. Accordingly, the Heliospectra was operated at 458 'intensity clicks' reading in the red $(666 \mathrm{~nm})$ power supply setting.

It was observed in prior preliminary experiments with capsicum plants, that if the LED light source is hung too close at approximately $0.6 \mathrm{~m}$ from the ground, that this caused the plants on the outer peripheral edges of the tent to bolt, due to not receiving enough light, and the plants within the range of the light to become yellow, due to receiving too much light. Therefore, it was determined for even spread of the light within the tent, to hang the LED light as high as possible within the constraints of the tent. This allowed the reflective sides of the tent to be utilized to the fullest effect. In addition, the wooden posts supporting the tent were kept as close to the edges as possible, to provide only minimal shading to the plants, to prevent bolting as much as possible.

\subsection{Power Consumption of LED Light Treatments}

The power consumed by the light treatment in each grow tent was measured using an off-the-shelf energy cost meter (Electus Distribution Pty Ltd., Rydalmere, NSW, Australia). The meter was used to directly quantify the power consumed $(\mathrm{W})$, as well as the voltages and currents required by the power supplies of the lighting appliances in each tent. The three lighting configurations were comparable (almost identical) in terms of power consumption, as outlined in Table 1.

Table 1. The power being consumed (W) for the light treatments in each grow tent.

\begin{tabular}{cc}
\hline Grow Tent Light Treatment & Power Consumption (W) \\
\hline White: Tent 1 & 245.2 \\
Red, blue: Tent 2 & 209 \\
Red, blue, far-red: Tent 3 & 256.8 \\
\hline
\end{tabular}

Therefore, using the more complex lighting appliance configuration employing an optimized triple-wavelength combination source has maintained essentially the same energy-efficiency as a common white light LED source. These data also indicate that future commercial greenhouse installations may also benefit from replacing conventional lighting appliances with optimized multi-wavelength LED sources. This would be in conjunction with passive thin-film filters, so as to shape and optimize the illumination spectra as required, throughout the day. 


\subsection{Plant Materials and Experimental Setup Details}

90 baby butterhead lettuce (Lactuca sativa L.) seedlings were sown individually in high quality seed and cutting potting mix in pots of $13 \mathrm{~cm}$ height. An additional sample of five seedlings was culled, dried, weighed, and averaged to obtain a zero-biomass starting point. The position of the plants within each tent was randomized every 7-8 days throughout the duration of the $39 \mathrm{~d}$ experiment. $50 \mathrm{~mL}$ of water was supplied by hand every day to each individual plant within each tent. Every 14 days, $50 \mathrm{~mL}$ of diluted liquid nutrient (Scotts Osmocote Plus Organics non-MU concentrate liquid fertilizer and soil improver diluted to approximately $0.06 \mathrm{~g}$ urea and approximately $0.04 \mathrm{~g}$ of other non-hazardous ingredients) was supplied to each plant, without any additional water [29].

The plants were illuminated inside each closed grow tent under different light treatments for an $18 \mathrm{~h}$ photoperiod. Grow Tent 1 received a light treatment of full power white light (1000 Heliospectra 'intensity' reading). Note: The 'intensity' of the Heliospectra LED light source is directly related to the power density, however, the 'intensity' is not actually a measurement in the units of intensity. Therefore, for the purpose of this paper, the 'intensity' when referring to the reading on the Heliospectra system, will be placed in inverted commas ("). Grow Tent 2 received a light treatment of full power blue $448 \mathrm{~nm}$ LED light (1000 Heliospectra 'intensity' reading), and approximately half power red $666 \mathrm{~nm}$ LED light (458 Heliospectra 'intensity' reading). Grow Tent 3 received a light treatment of full power blue $448 \mathrm{~nm}$ LED light (1000 Heliospectra 'intensity' reading), approximately half power red $666 \mathrm{~nm}$ LED light (458 Heliospectra 'intensity' reading), and full power far-red $736 \mathrm{~nm}$ LED light (1000 Heliospectra 'intensity' reading).

The 'intensity' values chosen model sunlight as closely as possible within the constraints of the Heliospectra LED light source. The LEDs were operated during the afternoon and evening from approximately 16:00-10:00 and off for six hours between approximately 10:00-16:00. The grow tents were substantially closed, except for panels with netting to allow air flow in, on three of four sides towards the base of the tent for ventilation. The fourth side was the entrance to the tent, and the zip was left open towards the base to further increase air flow and ventilation. The tents were opened daily to water each plant, and when randomizing the position of the plants. Otherwise, the tents remained essentially closed.

The parameters measured included the wet weight (g/plant), dry weight (g/plant) and biomass (g) of each plant. The photosynthetically active radiation (PAR) was measured via correlation to the power density for each light treatment, and the photosynthetic photon flux density $\left(\mu \mathrm{molm}^{-2} \mathrm{~s}^{-1}\right)$ was calculated. Figures of the growth results at different stages of the experiment are shown in the Supplementary Information, Figure S4.

Heliospectra LX602C LED light sources were used due to the availability of wavelength channels and the range of power output. Inside each grow tent, a Heliospectra LX602C LED light source was hung at $1.4 \mathrm{~m}$ above the top of each pot, where the pots were placed on the ground. Wood bracing was used to brace the structure of the tent to support the weight of the light source. The photon flux density (PFD) values determined from the calibration method outlined in the Supplementary Information are shown in Table 2.

Table 2. The normalized ratio of blue to red LED light and red to far-red LED light using the calculated photon flux density (PFD). Please note that the calculated PFD figure has been evaluated with less accuracy for the white light source, compared to other LED, due to the nature of this compound-source spectrum.

\begin{tabular}{cccc}
\hline Grow Tent & $\begin{array}{c}\text { Photon Flux } \\
\text { Density (PFD) }\end{array}$ & $\begin{array}{c}\text { Blue to Red Ratio } \\
\text { Normalized }\end{array}$ & $\begin{array}{c}\text { Red to Far-Red Ratio } \\
\text { Normalized }\end{array}$ \\
\hline $\begin{array}{c}\text { 1-White, } 1000 \text { 'intensity' } \\
\text { 2-Blue, } 1000 \text { 'intensity'; red, } 458 \\
\text { 'intensity' }\end{array}$ & $\sim 101 \mu \mathrm{molm}^{-2 \mathrm{~s}-1}$ (3 s.f.) & N/A & N/A \\
$\begin{array}{c}\text { 3-Blue, } 1000 \text { 'intensity'; red, } 458 \\
\text { 'intensity'; far-red, } 1000 \text { 'intensity' }\end{array}$ & $\sim 61.9 \mu \mathrm{molm}^{-2 \mathrm{~s}-1}$ (3 s.f.) & $1: 6.6$ & N/A \\
\hline
\end{tabular}


The first (White) control grow tent had a greater PFD than the third (blue, red, far-red) grow tent, yet the first (White) grow tent had the least biomass, and the third grow tent (blue, red, far-red) had the greatest biomass.

\section{Principal Results and Discussion}

Comparing data to that reported by other researchers, it was noted that Lee et al. [18] used various ratios of red to far-red LED light and blue LED light, however, the control for the experiment was a fluorescent lamp, not white LED. Therefore, the results could not be accurately compared to this paper [18], due to the difference in the controls and the blue to red, and red to far-red ratios being substantially different to that of the experiment conducted by ESRI. Lee et al. [18] used the ratios of blue to red 2:8, and red to far-red ratios of 0.7, 1.2, 4.1, 8.6. Han et al. [19] suggested that the ideal light for lettuce is a combination of red, yellow, and blue LED light. However, the comparison between the narrow white or broad white results, and the red, yellow, and blue results indicate that the biomass increase was about $1 / 3$ more in total weight (g) for the red, yellow and blue results. Both results in the broad white and narrow white appear (from the results shown in the article [19]) to be approximately equal. After 22 days, the dry total weight of plants in the red, yellow, blue LED light-treatment experiment was approximately $5 \mathrm{~g}$. Extrapolating to compare to our experimental results at 22 days (from the 0-39 days growth timeframe) indicates that the red, blue, far-red (RB, FR) results at 22 days would have been approximately $3.2 \mathrm{~g}$. Whilst this figure is less than that reported by Han et al. [19], it can also be noted that the red, yellow, blue results of Han et al. were receiving more normalized PFD, in comparison to the other light treatments. The PFD values of our experiment were greatest in the white light tent (which had the least biomass results), and the second-largest in the blue, red, and far-red tent (which actually had the greatest biomass results). It is important to note that biomass growth results have been reported to not necessarily vary linearly with photon flux density [30]. The results for the average fresh leaf weight, dry leaf weight, and biomass of the plants are shown in Table 3.

Table 3. The average fresh weight (FW), average dry weight (DW) and average biomass over the 90-plant sample.

\begin{tabular}{ccccc}
\hline \multirow{2}{*}{ Day } & LED Treatment & \multicolumn{2}{c}{ Leaf } \\
\cline { 3 - 5 } & & $\begin{array}{c}\text { Average FW } \\
\text { (g/plant) }\end{array}$ & $\begin{array}{c}\text { Average DW } \\
\text { (g/plant) }\end{array}$ & $\begin{array}{c}\text { Mean Biomass (g/plant); Standard } \\
\text { Deviation (g) }\end{array}$ \\
\hline 0-Zero biomass & N/A & 1.68 & 0.07 & 0.07 \\
& White & 83.45 & 4.91 & $4.84 ; 0.4156$ \\
& Blue + red & 84.55 & 5.22 & $5.15 ; 0.4853$ \\
& Blue + red + & 84.23 & 5.62 & $5.55 ; 0.3553$ \\
\hline
\end{tabular}

The raw data on the dry biomass obtained from each of the samples in each of the grow tents are shown in Figure 2, together with the graphical analysis of the relevant data distributions. The results indicate that the light condition of blue + red + far-red visible LED light provided the highest average dry weight and the highest average biomass, and the second-highest average fresh weight. The highest wet weight was produced under the blue and red visible LED light treatment. The white LED light treatment, which was used as the control, produced the lowest average fresh weight, the lowest average dry weight and the lowest average biomass. The T-tests were run (using standard Microsoft Excel functions providing a 2-sample array-based t-test evaluation for 2-tailed sample population distributions with unequal variance) using the dry weight data arrays for each pair of datasets. The numerical value outputs of these t-tests return the probability associated with a Student's t-test, which determines whether the two samples are likely to have come from the same two underlying populations that have the same mean. The $t$-test results obtained revealed evidence of a strong, statistically significant difference between the population means, in each of the tests. Numerically, the t-test results obtained from each pair of grow tents were: TTEST(T1,T2) $=0.0104$, $\operatorname{TTEST}(\mathrm{T} 1, \mathrm{~T} 3)=2.037 \times 10^{-9}$, and TTEST $(\mathrm{T} 2, \mathrm{~T} 3)=0.00057$. 


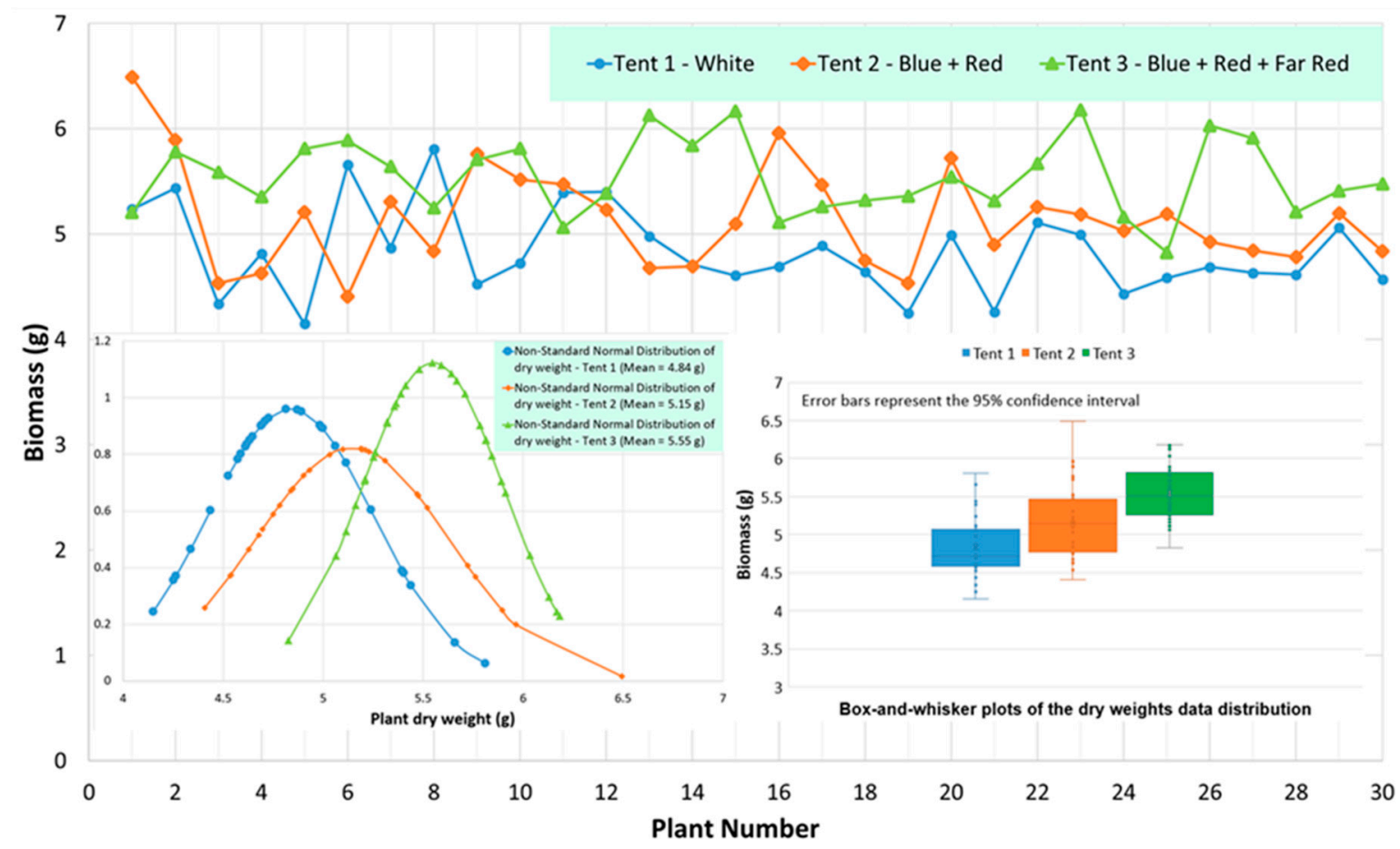

Figure 2. Graphical analysis of each of the grow tents, and the biomasses obtained by each plant within each tent (total of 90 plants).

The biomass results indicate that on comparison with the white (W) light treatment, the biomass in the blue + red + far-red light treatment was approximately $14.7 \%$ (3 s.f.) higher than the biomass in the $\mathrm{W}$ light treatment. Additionally, the biomass in the blue + red light treatment in comparison to the W light treatment, was approximately $6.41 \%$ (3 s.f.) higher than the $\mathrm{W}$ light treatment. The addition of far-red illumination source (adding approximately $8.7 \mu \mathrm{molm}^{-2} \mathrm{~s}^{-1}$ to the total PFD, which contributed an extra $14.05 \%$ to the total PFD) has led to a biomass increase of about $7.767 \%$, compared to using the blue and red light only. The light in the $\mathrm{W}$ light treatment was broader and received more PPF than the discrete light treatments in the blue + red, and the blue + red + far-red light treatments. Therefore, it could be hypothesized that the $\mathrm{W}$ control tent would have the greatest biomass. At the very least, the control group has not excluded any particular wavelength range, avoiding any accidental productivity reductions, due to excluding any known wavelengths of importance. The results, however, contradict this hypothesis. Even though it could have been anticipated that providing a greater amount of photon energy widely distributed across the spectrum would lead to generating greater biomass, it is also known that the light intensity response curve of photosynthesis is not flat.

The experimental results indicate that within the experiment, the wavelength range offering substantial biomass improvements for Lactuca sativa L., in comparison with the White LED control grow tent, are LED sources in the following spectral ranges: $448 \mathrm{~nm}$ (blue, FWHM 430-470 nm), and the range 666-736 nm (red, FWHM 650-678 nm, and far-red visible, FWHM 714-758 nm, respectively). As such, the narrow band central wavelength of $448 \mathrm{~nm}$ and the larger wavelength band of 666-736 nm were used to design the thin-film filter. All other wavelengths, that is, green (approximately 500-600 nm) visible, ultraviolet (UV approximately 10- approximately $400 \mathrm{~nm}$ ) and infrared (IR approximately 780-1000 $\mu \mathrm{m}$ [31]) were filtered.

\section{Thin-Film Filter for Future Solar Window Greenhouses}

As transmission characteristics of materials vary as a function of wavelength and type of material, it is necessary to choose materials that can provide the desired transmission characteristics when combined into layers [32,33]. A theoretical example having 18 layers and three materials $\left(\mathrm{MgF}_{2}, \mathrm{ZnS}\right.$, 
$\mathrm{Ag}$ ) is shown below, followed by a more practical fabrication example having nine layers with three materials $\left(\mathrm{Al}_{2} \mathrm{O}_{3}, \mathrm{ZnS}, \mathrm{Ag}\right)$.

The thin-film optical filter will ideally attenuate the waveband from $300 \mathrm{~nm}$ to $400 \mathrm{~nm}$, provide maximum transmission (allow the visible light to pass through) at $401-500 \mathrm{~nm}$ (blue), then suppress the waveband from $501 \mathrm{~nm}$ to $600 \mathrm{~nm}$ (green and yellow), and provide maximum transmission from $601 \mathrm{~nm}$ to $750 \mathrm{~nm}$ (red and far-red). This will provide the blue, red, and far-red visible ranges to be provided to the lettuce plants and ideally filter the wavelengths exterior to these ranges.

The thin-film optical filter was fabricated using three common optical materials: $\mathrm{Al}_{2} \mathrm{O}_{3}, \mathrm{ZnS}_{\text {, }}$ and Ag [34-38]. E-beam evaporation was used for the dielectric materials, and Ag was evaporated thermally within the same chamber. These techniques overcome the potential issues related to the possibility of target materials cross-contaminating during sputtering, as the plasma when sputtering fills the whole chamber and may cross-contaminate some of the target materials, as the shutters covering the materials do not fully seal the sources [39]. E-beam evaporation utilizes crucibles that are exposed to the chamber volume individually. These crucibles are rotated into position from closed to open. Additionally, the deposition of material layers can be more accurate compared to sputtering, since the quartz sensor (layer thickness monitor) is not affected by RF noise. The thin-film optical filter models and the corresponding fabrication results are discussed in the following sections.

The first design was an 18-layer thin-film filter designed using OptiLayer Pro with a single $14.5 \mathrm{~nm}$-thick Ag layer. The dielectric materials utilized were $\mathrm{MgF}_{2}$, and $\mathrm{ZnS}$. The results of the simulation are shown in Figure 3. An 18 layer design is typically time-consuming and costly to fabricate, and is described here only for illustration purposes. Accordingly, an alternative filter using only nine layers and the common materials $\mathrm{Al}_{2} \mathrm{O}_{3}, \mathrm{ZnS}$ and $\mathrm{Ag}$ was designed, and fabricated.

The fabrication conditions of the nine-layered thin film filter were:

Tooling Factor (TF) of $\mathrm{ZnS}, \mathrm{Al}_{2} \mathrm{O}_{3}$, and $\mathrm{Ag}$ were $77 \%, 95 \%$, and $140 \%$, respectively. The total desired thickness was $322 \mathrm{~nm}$ which was comprised of a symmetrical design, having $\mathrm{Al}_{2} \mathrm{O}_{3}$ outer layers, followed by alternating layers of $\mathrm{ZnS}, \mathrm{Ag}, \mathrm{ZnS}$, with a central layer of $\mathrm{Al}_{2} \mathrm{O}_{3}$ and again followed by alternating layers of $\mathrm{ZnS}, \mathrm{Ag}$ and $\mathrm{ZnS}$. The desired thicknesses were also symmetrical around the central $\mathrm{Al}_{2} \mathrm{O}_{3}$ layer, and equivalent for the layers that were of the same material.

The comparison results of the measured data compared to the modelled data for the nine-layered thin-film filter design are shown in Figure 4. The low-emissivity transmission spectrum-shaping filter presented in Figure 5 will replace the current heat-mirror films employed in ClearVue solar windows [40], which will be installed into a pilot solar greenhouse. Figure 5a illustrates a photograph of the thin-film filter filtering sunlight through a coated glass window. As can be observed, the green part of the visible spectrum is suppressed, as required, whilst the blue, red, and far-red components of the visible spectrum are being transmitted to the plants below. Note that, by using a higher number of layers, thin-film optical filters, which exhibit better correlation to the model in the green range of the visible spectrum, can be developed. 


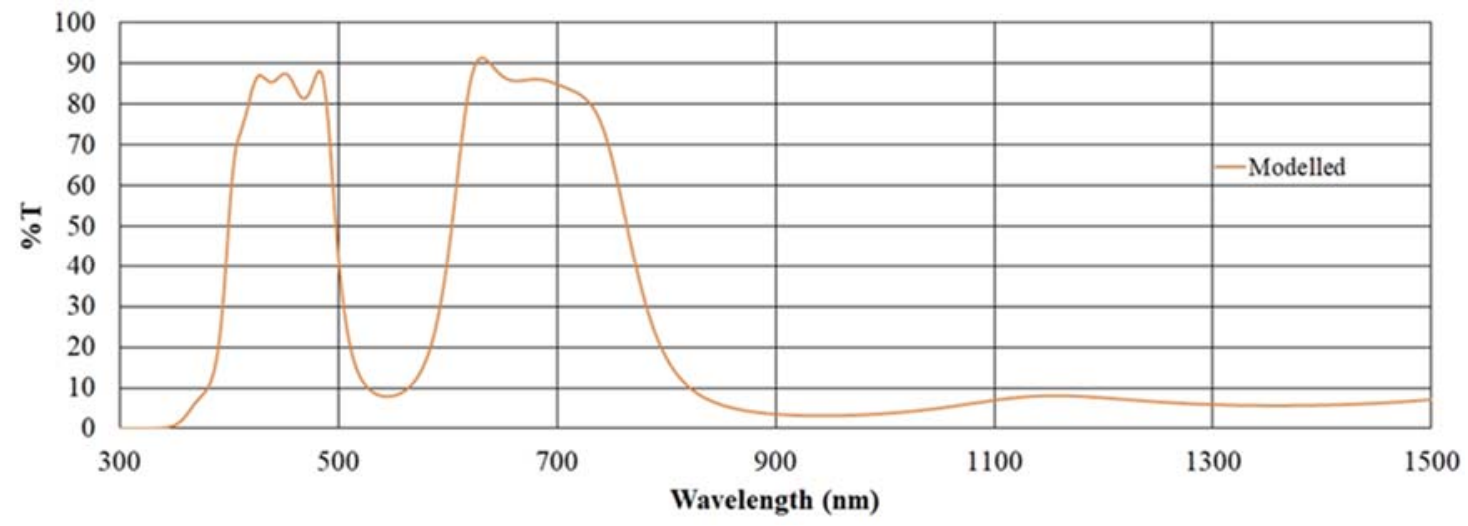

Figure 3. Simulated plot data from Opti-Layer Pro for an 18 layer thin-film filter design using $\mathrm{MgF}_{2}$, $\mathrm{ZnS}$ and $\mathrm{Ag}$.

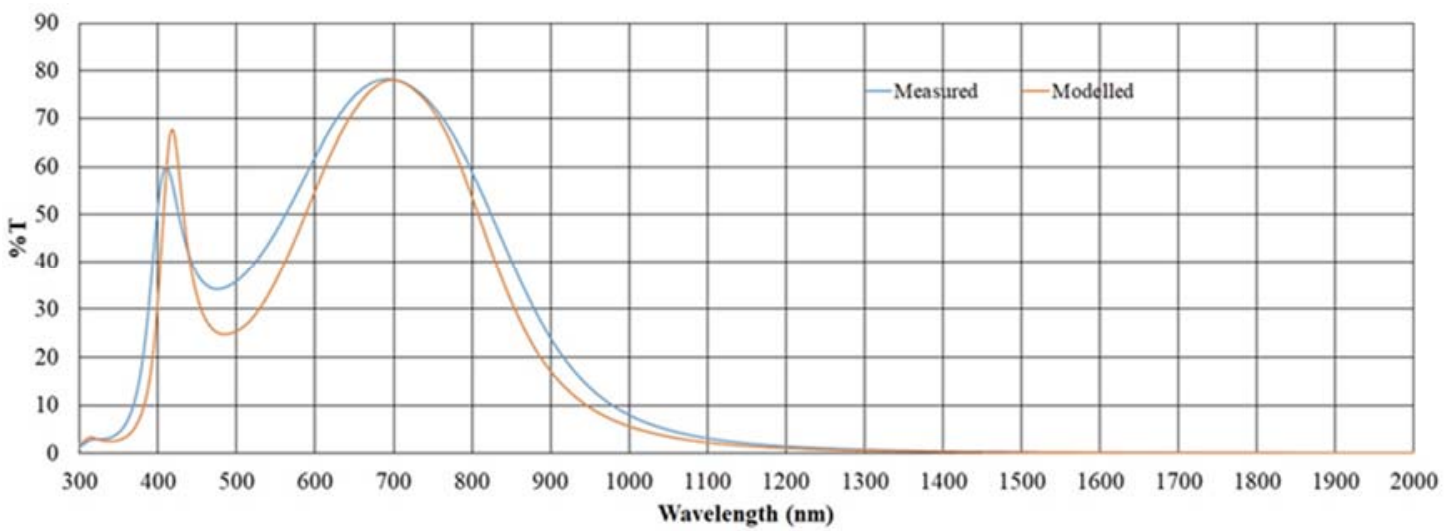

Figure 4. Simulated plot data from Opti-Layer Pro for a nine-layered thin-film filter design using $\mathrm{Al}_{2} \mathrm{O}_{3}, \mathrm{ZnS}$ and $\mathrm{Ag}$, plotted with the transmission results obtained from a UV/Vis Spectrophotometer.

The design and manufacturing techniques used to prototype the 9-layer filter design of Figure 4 were adopted from ESRI's extensive prior experience with double-silver low-emissivity film designs, possessing high environmental exposure stability [41]. Figure 5 shows the visual appearance and color properties of a thin-film filter sample prototyped at ESRI, ECU, and also illustrates the spectral modification of natural sunlight's spectral contents, occurring on a normal-incidence transmission through this filter, in comparison with the spectral effects of a 6mm-thick ultraclear low-iron uncoated glass.

As distinct from all of the commercially fabricated low-emissivity double-silver film samples on glass substrates trialed by ESRI to date, the metal-dielectric designs (including that of Figure 4; Figure 5) can withstand exposure to moisture, water, and cleaning solvents. Figure 6 shows the environmental exposure stability test results, obtained using an air-filled laboratory box-furnace oven and an environmental test chamber. 

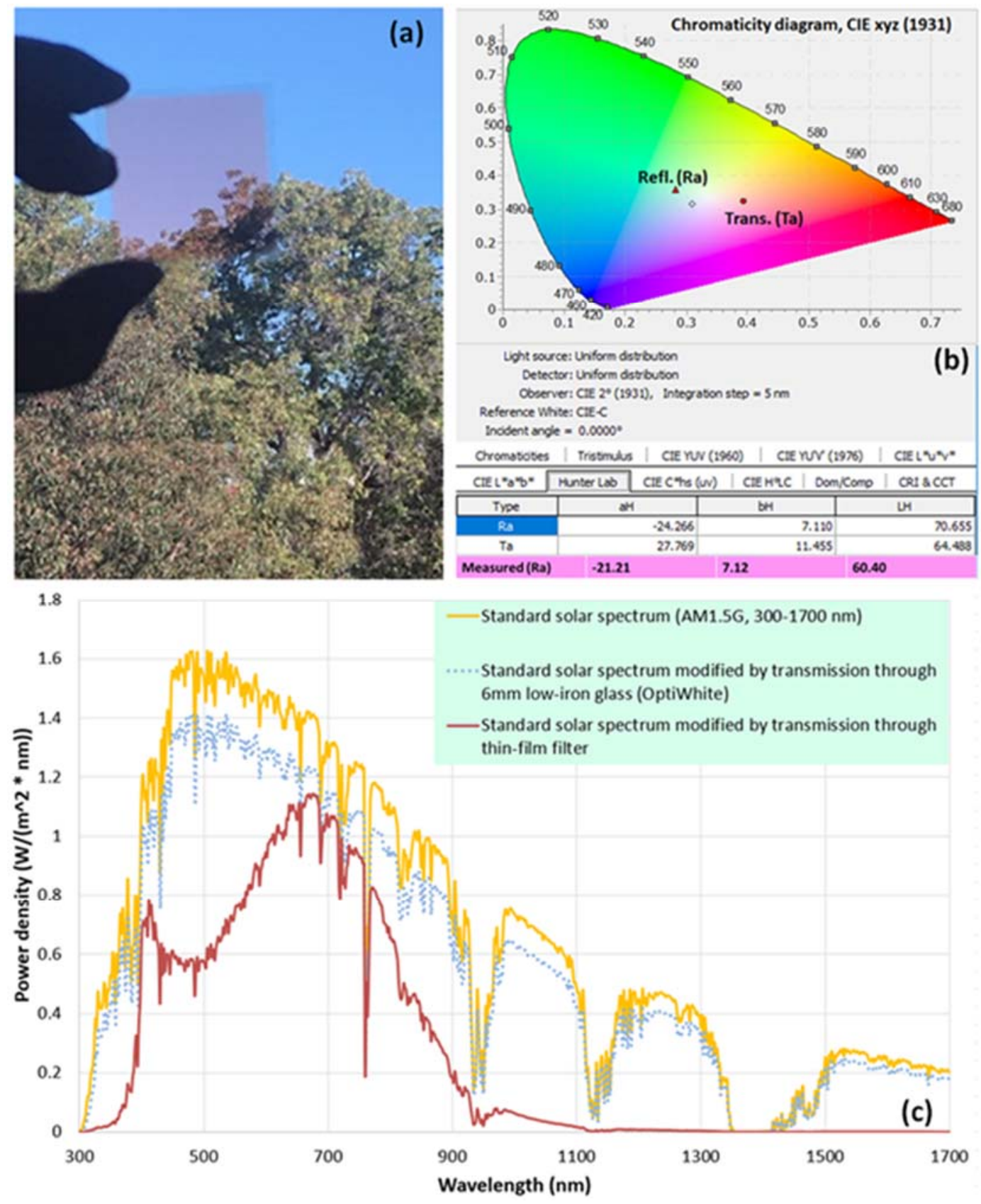

Figure 5. Thin-film filter on a glass substrate modifying the incoming natural sunlight. (a) filter image taken with the sample placed next to the glass of a conventional window; (b) filter chromaticity diagram, modeled in transmission and reflection; the measured reflected-color Hunter Lab parameters (L, a, b) are also shown; (c) spectral modification of the natural sunlight (standard AM1.5G spectrum) on transmission through either the low-iron ultraclear $6 \mathrm{~mm}$ glass, or through the thin-film Figure 4 . 

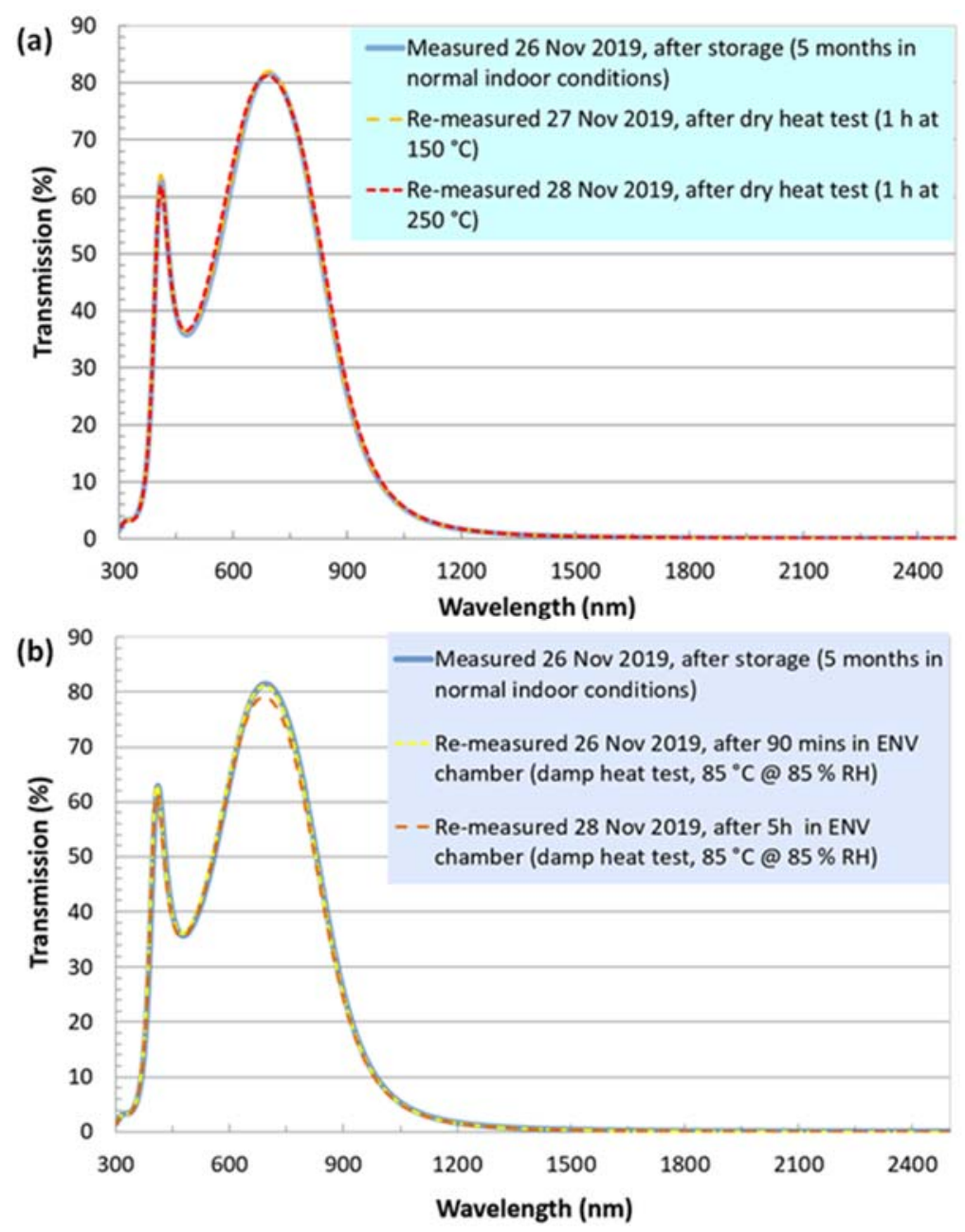

Figure 6. Environmental exposure test results obtained with the thin-film filter of Figure 5a, demonstrated by the stability of filter transmission characteristics. (a) dry heat exposure test results; (b) damp heat test results.

The following sequences of testing procedures were performed with the separately tested two glasscutter-separated parts of the filter sample shown in Figure 6a: (i) First dry heat exposure test, conducted at $150{ }^{\circ} \mathrm{C}$, followed by another dry heat exposure at $250{ }^{\circ} \mathrm{C}$, applied to the first pre-cut part of the sample; (ii) first damp heat test run for 90 mins, followed by another damp heat test run for $5 \mathrm{~h}$, using the second pre-cut part of the same sample. No visible signs of sample surface degradation have been observed, and only minute spectral property changes were detected.

These features of double-silver metal-dielectric coatings on glass substrates will enable safe transport of future advanced greenhouse coating products from the coating manufacturing facilities, to the glass industry assembly factories, where the production of specific solar window types will take place. A unique combination of advanced glazing system features can therefore be realized-including the low thermal emissivity, heat shielding properties, custom-shaped transmitted color and spectrum, and the suitability for use as components in photovoltaic energy-harvesting window designs.

\section{Conclusions}

A wavelength range of visible light can be optimized for obtaining improved biomass growth results from the sample plant Lactuca sativa, L. using energy-efficient LED light sources. Furthermore, dry biomass improvements in excess of $14 \%$ (compared to results obtained with white LED illumination) have been demonstrated in growth tent experiments that utilize a spectrally-optimized 
combination of LED illumination sources. Experimental results have confirmed the importance of providing blue, red, and far-red visible wavelengths to plants for biomass productivity improvements. Even though several prior studies have also reported on the importance of these wavelength ranges for promoting plant growth productivity, our experiments have re-confirmed obtaining improved biomass production at relatively low optical power densities available from energy-efficient optical sources (compared to natural sunlight at weather conditions close to the NREL AM1.5G standard). These results have laid the foundation for the development of a prototype of a passive solar thin-film filter design suitable for use in existing low-emissivity energy-harvesting solar window products, for use in agricultural greenhouses. It can be expected that future lettuce growth experiments in advanced pilot greenhouses, which also use spectrally optimized solar windows, will reconfirm the biomass improvement results reported.

Supplementary Materials: The following are available online at http://www.mdpi.com/2071-1050/12/9/3740/s1, Figure S1: Schematic diagram of the Heliospectra LED source calibration setup, Figure S2: The power density calibration plots of the Heliospectra LED source, Figure S3: The photon flux density calibration plots of the Heliospectra LED source, Figure S4: Growth of a randomly selected plant, the same plant number within each of the three different illuminated grow tents. (a-c) Grow Tent 1, Plant 8 growth from (a) day 12, (b) day 22 and (c) day 34. (d-f) Grow Tent 2, Plant 8 growth from (d) day 12, (e) day 23 and (f) day 34. (g-i) Grow Table 3. Plant 8 growth from (g) day 13, (h) day 23, and (i) day 34 .

Author Contributions: Conceptualization, J.A.T., M.V. and K.A.; methodology, J.A.T., M.V., M.N.-E.-A. and K.A.; software, J.A.T., M.V. and M.N.-E.-A.; validation, J.A.T., M.V. M.N.-E.-A. and K.A.; formal analysis, J.A.T., M.V. M.N.-E.-A. and K.A.; investigation, J.A.T., M.V. and K.A.; resources, J.A.T., M.V. M.N.-E.-A. and K.A.; data curation, J.A.T., and M.V. and K.A.; writing-original draft preparation, J.A.T., M.V.; writing-review and editing, J.A.T., M.V. M.N.-E.-A. and K.A.; visualization, J.A.T., M.V. and K.A.; supervision, M.V. M.N.-E.-A. and K.A.; project administration, K.A.; funding acquisition, K.A. All authors have read and agreed to the published version of the manuscript.

Funding: This research was funded by the Australian Government through a Cooperative Research Centre Project and Edith Cowan University.

Acknowledgments: The authors would like to acknowledge the fruitful discussions and valuable inputs provided by W. Lonsdale, resulting in the improvements of the thin-film deposition process.

Conflicts of Interest: The authors declare no conflict of interest. The funders had no role in the design of the study; in the collection, analyses, or interpretation of data; in the writing of the manuscript, or in the decision to publish the results.

\section{References}

1. Alexandratos, N.; Bruinsma, J. World Agriculture towards 2030/2050; FAO: Rome, Italy, 2012; p. 375.

2. Lancet, T. 2019: The year for nutrition. Lancet 2019, 393, 200. [CrossRef]

3. Willett, W.; Rockström, J.; Loken, B.; Springmann, M.; Lang, T.; Vermeulen, S.; Garnett, T.; Tilman, D.; Declerck, F.; Wood, A.; et al. Food in the Anthropocene: The EAT-Lancet Commission on healthy diets from sustainable food systems. Lancet 2019, 393, 447-492. [CrossRef]

4. Tosti, G.; Benincasa, P.; Cortona, R.; Falcinelli, B.; Farneselli, M.; Guiducci, M.; Onofri, A.; Pannacci, E.; Tei, F.; Giulietti, M. Growing lettuce under multispectral light-emitting diodes lamps with adjustable light intensity. Ital. J. Agron. 2017, 11, 57-62. [CrossRef]

5. Zhang, T.; Shi, Y.; Wang, Y.; Liu, Y.; Zhao, W.; Piao, F.; Sun, Z. The effect of different spectral LED lights on the phenotypic and physiological characteristics of lettuce (Lactuca sativa) at picking stage. J. Biochem. Biotechnol. 2017, 1, 14-19. [CrossRef]

6. Chang, C.-L.; Chang, K.-P. The growth response of leaf lettuce at different stages to multiple wavelength-band light-emitting diode lighting. Sci. Hortic. 2014, 179, 78-84. [CrossRef]

7. Kang, J.H.; Krishnakumar, S.; Atulba, S.L.S.; Jeong, B.R.; Hwang, S.J. Light intensity and photoperiod influence the growth and development of hydroponically grown leaf lettuce in a closed-type plant factory system. Hortic. Environ. Biotechnol. 2013, 54, 501-509. [CrossRef]

8. Kim, H.-H.; Goins, G.D.; Wheeler, R.M.; Sager, J.C. Green-light Supplementation for Enhanced Lettuce Growth under Red- and Blue-light-emitting Diodes. HortScience 2004, 39, 1617-1622. [CrossRef] 
9. Salisbury, F.B.; Ross, C.W. Plant Physiology, Hormones and Plant Regulators: Auxins and Gibberellins, 4th ed.; Wadsworth Publishing: Belmont, CA, USA, 1992; pp. 357-381.

10. Singh, D.; Basu, C.; Meinhardt-Wollweber, M.; Roth, B.W. LEDs for energy efficient greenhouse lighting. Renew. Sustain. Energy Rev. 2015, 49, 139-147. [CrossRef]

11. Corrado, C.; Leow, S.W.; Osborn, M.; Carbone, I.; Hellier, K.; Short, M.; Alers, G.; Carter, S.A. Power generation study of luminescent solar concentrator greenhouse. J. Renew. Sustain. Energy 2016, 8, 043502. [CrossRef]

12. Emmott, C.J.M.; Rohr, J.; Campoy-Quiles, M.; Kirchartz, T.; Urbina, A.; Ekins-Daukes, N.; Nelson, J. Organic photovoltaic greenhouses: A unique application for semi-transparent PV? Energy Environ. Sci. 2015, 8, 1317-1328. [CrossRef]

13. Marondedze, C.; Liu, X.; Huang, S.; Wong, C.; Zhou, X.; Pan, X.; An, H.; Xu, N.; Tian, X.; Wong, A. Towards a tailored indoor horticulture: A functional genomics guided phenotypic approach. Hortic. Res. 2018, 5, 68. [CrossRef] [PubMed]

14. Silva, E.; Maluf, W.R.; Leal, N.; Gomes, L. Inheritance of bolting tendency in lettuce Lactuca sativa L. Euphytica 1999, 109, 1-7. [CrossRef]

15. LoikiD, M.E.; Carter, S.A.; Alers, G.; Wade, C.E.; Shugar, D.; Corrado, C.; Jokerst, D.; Kitayama, C. Wavelength-Selective Solar Photovoltaic Systems: Powering Greenhouses for Plant Growth at the Food-Energy-Water Nexus. Earth's Futur. 2017, 5, 1044-1053. [CrossRef]

16. Vasiliev, M.; AlGhamedi, R.; Nur-E-Alam, M.; Alameh, K. Photonic microstructures for energy-generating clear glass and net-zero energy buildings. Sci. Rep. 2016, 6, 31831. [CrossRef] [PubMed]

17. Vasiliev, M.; Alameh, K.; Nur-E-Alam, M. Spectrally-Selective Energy-Harvesting Solar Windows for Public Infrastructure Applications. Appl. Sci. 2018, 8, 849. [CrossRef]

18. Lee, M.-J.; Son, K.-H.; Oh, M.-M. Increase in biomass and bioactive compounds in lettuce under various ratios of red to far-red LED light supplemented with blue LED light. Hortic. Environ. Biotechnol. 2016, 57, 139-147. [CrossRef]

19. Han, T.; Vaganov, V.; Cao, S.; Li, Q.; Li-Li, L.; Cheng, X.; Peng, L.; Zhang, C.; Yakovlev, A.N.; Zhong, Y.; et al. Improving "color rendering" of LED lighting for the growth of lettuce. Sci. Rep. 2017, 7, 45944. [CrossRef]

20. Meng, Q.; Runkle, E.S. Far Red is the New Red. Inside Grow. 2017, pp. 26-30. Available online: https: //www.canr.msu.edu/floriculture/uploads/files/far-red-on-lettuce.pdf (accessed on 27 April 2020).

21. Lin, K.-H.; Huang, M.-Y.; Huang, W.-D.; Hsu, M.-H.; Yang, Z.-W.; Yang, C.-M. The effects of red, blue, and white light-emitting diodes on the growth, development, and edible quality of hydroponically grown lettuce (Lactuca sativa L. var. capitata). Sci. Hortic. 2013, 150, 86-91. [CrossRef]

22. McCree, K. The action spectrum, absorptance and quantum yield of photosynthesis in crop plants. Agric. Meteorol. 1971, 9, 191-216. [CrossRef]

23. Nelson, J.A.; Bugbee, B. Economic Analysis of Greenhouse Lighting: Light Emitting Diodes vs. High Intensity Discharge Fixtures. PLoS ONE 2014, 9, e99010. [CrossRef]

24. Darko, E.; Heydarizadeh, P.; Schoefs, B.; Sabzalian, M.R. Photosynthesis under artificial light: The shift in primary and secondary metabolism. Philos. Trans. R. Soc. B Boil. Sci. 2014, 369, 20130243. [CrossRef] [PubMed]

25. Lu, N.; Maruo, T.; Johkan, M.; Hohjo, M.; Tsukagoshi, S.; Ito, Y.; Ichimura, T.; Shinohara, Y. Effects of Supplemental Lighting with Light-Emitting Diodes (LEDs) on Tomato Yield and Quality of Single-Truss Tomato Plants Grown at High Planting Density. Environ. Control. Boil. 2012, 50, 63-74. [CrossRef]

26. NREL U.S. Department of Energy. Reference Air Mass 1.5 Spectra. Available online: https://www.nrel.gov/ grid/solar-resource/spectra-am1.5.html (accessed on 8 March 2019).

27. RNG International Inc. Average Peak Sun Hours by State. Available online: https://www.renogy.com/ template/files/Average-Peak-Sun-hours-by-State.pdf (accessed on 8 March 2019).

28. Stapleton, G.; Neill, S.; Milne, G. Photovoltaic Systems. Your Home Australia's Guide to Environmentally Sustainable Homes. Available online: http://www.yourhome.gov.au/energy/photovoltaic-systems (accessed on 8 March 2019).

29. Safety, M.; Sheet, D.; Oil, W.M. Scotts Osmocote + Organics non MU Concentrate Datasheet. 2017, pp. 1-5. Available online: https://www.scottsaustralia.com.au/safety-data-sheets/ (accessed on 29 April 2020).

30. Van Henten, E. Validation of a dynamic lettuce growth model for greenhouse climate control. Agric. Syst. 1994, 45, 55-72. [CrossRef]

31. Giancoli, D.C. Physics: Prentice Hall, 1998, 5th ed.; Pearson Higher Education: Harlow, UK, 1998. 
32. Vaclavik, J.; Vápenka, D. Gallium Phosphide as a material for visible and infrared optics. EPJ Web Conf. 2013, 48, 28. [CrossRef]

33. Mao, K.; Shen, W.; Yang, C.; Fang, X.; Yuan, W.; Zhang, Y.; Liu, X. Angle Insensitive Color Filters in Transmission Covering the Visible Region. Sci. Rep. 2016, 6, 19289. [CrossRef]

34. Lin, Y.C.; Chen, Z.A.; Shen, C.H. Novel optical thin film color filter: Simulation and experiment. Chin. J. Phys. 2012, 50, 643-651.

35. Koseki, T.; Fukunaga, T.; Yamanaka, H.; Ueki, T. Color filter for 10.4-in.-diagonal 4096-color thin-film-transistor liquid crystal displays. IBM J. Res. Dev. 1992, 36, 43-50. [CrossRef]

36. Sugiura, T.; Sawada, T.; Tani, M.; Sakagawa, M. Development of pigment dispersed typecolor filters for LCDs. J. Soc. Inf. Disp. 1992, 23, 21-24.

37. Mizuno, K.; Okazaki, S. Printing Color Filter for Active Matrix Liquid-Crystal Display Color Filter. Jpn. J. Appl. Phys. 1991, 30, 3313-3317. [CrossRef]

38. Sabnis, R.W. Color filter technology for liquid crystal displays. Displays 1999, 20, 119-129. [CrossRef]

39. Ohring, M. Materials Science of Thin Films—Deposition \& Structure; Academic Press: Cambridge, MA, USA, 1991.

40. Vasiliev, M.; Nur-E-Alam, M.; Alameh, K. Recent Developments in Solar Energy-Harvesting Technologies for Building Integration and Distributed Energy Generation. Energies 2019, 12, 1080. [CrossRef]

41. Mouedden, Y.; Alghamedi, R.; Nur-e-Alam, M.; Vasiliev, M.; Alameh, K. Thin film coatings for solar and thermal radiation control prepared by physical vapour deposition. In Proceedings of the 20129 th International Conference on High Capacity Optical Networks and Enabling Technologies (HONET), Istanbul, Turkey, 12-14 December 2012; pp. 83-86.

(C) 2020 by the authors. Licensee MDPI, Basel, Switzerland. This article is an open access article distributed under the terms and conditions of the Creative Commons Attribution (CC BY) license (http://creativecommons.org/licenses/by/4.0/). 